\title{
Capillary Model of Gravity Elution of High Viscosity Liquid from a Porous Bed with a Low-Viscosity Liquid
}

\author{
Mariola M. Błaszczyk • Jerzy P. Sẹk
}

Received: 26 March 2014 / Accepted: 12 July 2014 / Published online: 28 August 2014

(C) The Author(s) 2014. This article is published with open access at Springerlink.com

\begin{abstract}
Modeling of the processes of elution from porous systems is essential importance for development of the removing oily contaminations from the soils and intensification of the oil recovery processes. In the paper, capillary model of gravitational elution of high viscosity substances from the porous medium by using low viscosity liquids was derived. This model allowed for the prediction of changes in time of such parameters like: level of bed saturation with oil, relative bed permeability, liquid flow rate, flow resistance, volume of eluted liquid during the process. When modeling, phenomena and the physical properties associated with the analyzed process, such as, for example, the effects of surface tension, fluids viscosity, specific size of the granular bed, initial oil saturation of bed, variable process driving force, and the flow of liquid through the preferential flow paths, were taken into account. This allowed for the more complete imaging of elution process. The model has been verified on the basis of the results of experimental studies. In addition, the discussion on the behavior of the model due to changes in values of various parameters was carried out.
\end{abstract}

Keywords Porous media $\cdot$ Capillary model $\cdot$ Elution

\section{Introduction}

The study of flows at multiphase systems of liquid-liquid type through porous structures is one of the important branches of process engineering. These issues can be the basis for the analysis of the phenomena of migration of petroleum substances in porous soil structures. Data on the intensity of these processes as well as their range are important for the development of

M. M. Błaszczyk $(\varangle) \cdot$ J. P. Sęk

Department of Chemical Engineering, Faculty of Process and Environmental Engineering,

Lodz University of Technology, Lodz, Poland

e-mail: mar.blaszczyk@o2.pl

J. P. Sęk

e-mail: jerzysek@p.lodz.pl 
soil remediation technology and protection of drinking water sources against dangerous for health contamination with substances often having a carcinogenic effect (Kajdas 1979).

It can be also remarked that the issue of flow of liquid-liquid systems in porous layers can be very important for the intensification of oil extraction processes from rock structures. The extraction of oil from previously economically unprofitable sources or from new areas such as oil sands or oil shale is of great importance for the world economy (Yan et al. 1999).

The flow of liquid-liquid systems through porous structures can be very often analyzed as an elution of highly viscous organic liquids such as crude oil or its derivatives by means of a low viscosity liquid-usually water. In the literature about these types of processes most frequently, a classical approach is adopted resulting from the application of Darcy's law and the concept of porous bed permeability to describe the above phenomena. There are empirical models based on predicting changes in the bed permeability relative to its saturation with one- or multi-phase systems (Fulcher et al. 1985; Bedient et al. 1994; Brooks and Cory 1966; Genuchten 1980). These equations usually do not take into account the characteristics of the porous bed or the physicochemical properties of flowing liquids.

Another approach to modeling flows through porous beds is the use of the concept introduced by Kozeny-Carman (Carman 1937; Kembłowski et al. 1985). According to this idea the porous bed is represented as a bundle of tortuous capillaries through which flow takes place. This approach is used to model one- and multi-phase flows. Here the impact of the properties of bed and flowing liquids on flow mechanisms is taken into account. This concept can be also applied for predicting of elution processes.

Replacing a high-viscosity fluid with a lower viscosity fluid in a capillary is a complicated and complex phenomenon. It can be divided into two cases of flows. The first one concerns the situation when two fluids flowing through the capillary tube are mixing together, and the second one occurs when such fluids do not exhibit the mutual mixing with each other. During miscible flow characterization, the diffusion phenomena between the fluids should be taken into account. However, in the immiscible flow only the dynamics of flowing liquids are considered. The phenomena accompanying miscible flow in a single channel were discussed and supported by relevant numerical simulations provided by Sahu et al. (2009a, b), Talon and Meiburg (2011), Talon et al. (2013), Taghavi et al. (2012). The various systems of channels with different initial conditions were analyzed there. The immiscible flows were described by Redapangu et al. $(2012,2013)$. The extensive review of instabilities in the above-mentioned systems is given in Govindarajan and Sahu (2014).

An extensive analysis of the phenomena of interaction between immiscible liquids in simple systems of two connected capillaries with different diameters was presented by Dullien (1992). These studies have involved the injection of walls wetting liquid into the capillaries which at the beginning were filled with a non-wetting liquid.

Also a more complex model of elution from capillary systems was proposed by Nutt (1982). The bases for developing this model were the considerations relating to the system of two parallel capillaries of the same length but of different diameters. The capillaries were initially completely filled with non-wetting liquid—oil which was then replaced by a wetting liquid-water. These considerations were extended by Nutt to bundles of many capillaries of different diameters and different diameter distributions.

The concept of eluting one of the liquids by the other in many parallel capillaries of different diameters was also used in the work presented by Yang et al. (2009). The authors accepted that inside capillaries flow may be described by the equation of Hagen-Poiseuille, taking into account the impact of the curvature of the boundary between the phases on the flow conditions. 
Idorenyin and Shirif (2012) also analyzed the case which was geometrically similar to the previous ones. On the basis of model calculations, the authors determined the velocity at the phase boundary shift in the individual capillaries. There was also examined the influence of such factors as intensity of liquid injection, ratio of the viscosity of liquids, the effect of surface tension and wettability.

Different aspects of a liquid flow process, using the concept of parallel capillary bundles, are discussed in the works of Wang and Dong (2011), Dahle et al. (2005), Aranyosi (2010), Wielhorski et al. (2012). In these works, the influence on the flow of such parameters like: shape of capillaries, their length, and different ways of their connections has been taken into account.

These discussed issues referred to the models in which the intensity of the inlet flow of liquid or pressure at the inlet had steady state values. However, there are many real cases, such as elution of oil pollution by rain or flood water with falling waterhead over time. Then, the driving force values resulting from the height of the liquid column above the granular bed are changing over time. In such situations, models presented in the literature based on the assumption of constant pressure and flow rate at the inlet can not be applied to the description of the real elution processes of highly viscous substances from the granular bed.

A great disadvantage of previously developed models based on the concept of bundles of capillaries is that there they do not provide relationship enabling the conversion of the actual granular medium into the bundle of capillaries of different diameters. The specified distribution of capillary diameter is adopted arbitrarily without any relation to the given structure of the bed. This does not allow to apply of such models to the specific flow cases in real existing structures.

Also most of the developed capillary models analyzes only the rate of movement of the interface between liquids in the given capillaries groups. However, there are no considerations concerning the flow when the oil was eluted entirely from a part of the capillaries and in the remaining part of capillaries this phenomenon continues. Therefore, it is impossible to follow, using existing approaches, the oil elution process during its total duration.

Models based on the concept of bundles of capillaries, described in the literature (Dullien 1992; Nutt 1982; Yang et al. 2009; Idorenyin and Shirif 2012) do not cover the phenomenon of liquid flow through preferential paths that takes place in the real porous media. As a result the reflection of existing real cases is considerably limited. In addition, most capillary models initially assume complete saturation of the bed with non-wetting liquid. However, in real situations there are frequently situations when the bed is filled initially with the highly viscous substance only to a certain degree.

In view of the limitations of the models presented in the literature, which are based on the concept of capillary bundles, in the present study there is presented a gravity driven capillary model developed for the elution of highly viscous liquid from granular bed by means of a low viscosity liquid at unsteady driving force of the process. Also the conditions determining the flow through preferential flow paths and determining the initial incomplete saturation of the bed with oil were taken into consideration. Furthermore, the procedure of conversion of definite granular bed at a given height and diameter, consisting of the grains of the given diameter distributions to a bundle of capillaries with corresponding diameter distributions, will be presented. This will allow to follow the elution process of the highly viscous substance from the bed in form of a granular layer of defined height and diameter. In result of the analyses, it will be possible to follow the changes in the oil saturation level, relative bed permeability, liquid flow rate, flow resistance, volume of eluted liquid during the process in relation to the unsteady, decreasing driving force. 


\section{Model Considerations}

The aim of the works to develop model was to present the relationships which could enable predicting changes of the liquid flow rate through a porous medium and changes of saturation as a function of time. Granular bed will be treated as a bundle of tortuous capillaries. Let us consider the case when the liquid of high viscosity — oil is removed from the granular layer by means of a low viscosity liquid-water.

In the first step of the process modeling we will discuss oil elution with water from a single straight capillary. Physical phenomena governing the process will be determined, which will enable the derivation of flow equations. The elution process will be also described in a situation when oil has flown out from the capillary and only water will flow through it.

In the next phase of modeling there will be discussed the interaction between the flows in connected, parallel capillaries of different diameters. In such a system a different elution degree can be expected in different capillaries at a time. The final model works will analyze the case of oil elution by water from a bundle of capillaries representing the granular bed as a column of a given diameter and height.

\subsection{Oil Gravity Elution from a Single Capillary}

Let us consider a vertical capillary of a height equal to $L$ and radius $r$ as shown in Fig. 1a. The capillary tube is filled to the height of $L_{0}$ with oil-a high viscosity liquid. Above the oil layer there is a low viscosity liquid - water. The total height of the layer of water is equal to $h$, and is the sum of two heights, as shown in Fig. 1a. The first one is the height of water column $h_{\mathrm{w}}$ in the capillary, and the other $H$, is the height of the column above the entrance to the capillary. According to this $h$ value can be expressed by the formula:

$$
H+h_{\mathrm{w}}=h=\mathrm{const}
$$

At the initial moment the height $H=H_{0}$. During the process of oil elution from the capillary value $H$ decreases and the value $h_{\mathrm{w}}$ increases. The value $h$ remains constant until the entire oil flows out of the capillary.

During flow the height $L_{\mathrm{o}}$ of the oil in the capillary decreases to zero and the value $h_{\mathrm{w}}$ increases to the value $L$. Therefore it can be expressed as:

$$
L_{\mathrm{o}}+h_{\mathrm{w}}=L=\text { const }
$$

The flow in the capillary occurs due to the interaction of the forces of gravity, capillary forces and viscous forces in the laminar regime of flow. A case where the oil during elution does not remain on the walls of capillaries is assumed. It means that elution takes place through the whole cross-section. According to the designations shown in Fig. 1a the value of pressure resulting from gravitational forces relative to the outlet of the capillary is:

$$
p_{\mathrm{G}}=\rho_{\mathrm{w}} g h+\rho_{\mathrm{o}} g l_{\mathrm{o}},
$$

where $\rho_{\mathrm{w}}$ is density of low viscosity liquid, water, $\rho_{\mathrm{o}}$ is density of high viscosity liquid, oil, $g$ is gravity acceleration.

The capillary forces result from the interaction of cohesion and adhesion forces between the molecules of liquids relative to each other and to the molecules of the surrounding material. Assuming that the capillary has a strong wettability property, water will go up the capillary walls and will displace oil. This means that the capillary pressure will act toward the interior of the capillary and thus will assist the process of flow. This phenomenon is true only in the case when the non-wetting liquid is replaced by wetting liquid, in the opposite case, the 
Fig. 1 Gravitational oil elution from a single, vertical capillary

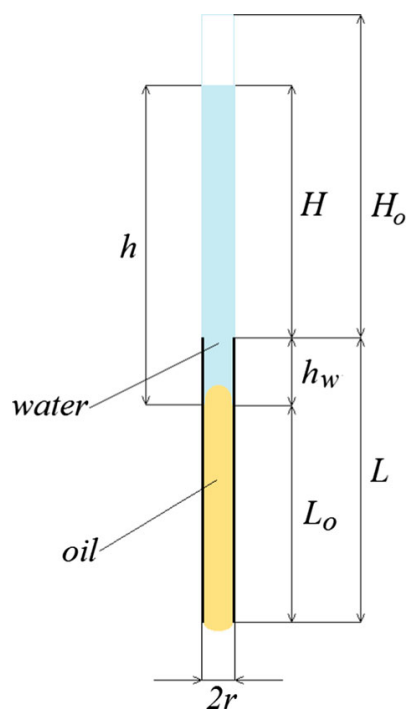

capillary force will oppose the flow. At the end of the capillary, where oil is bordered with air, the capillary force will have the opposite direction, which will decelerate the flow (Aranyosi 2010; Ahmadlouydarab et al. 2011).

Generally, the pressure caused by the capillary forces in a cylindrical capillary of radius $r$ can be expressed as:

$$
p_{\mathrm{C}}=\frac{2 \sigma_{\mathrm{ow}} \cos \theta_{\mathrm{ow}}}{r}-\frac{2 \sigma_{\mathrm{oa}} \cos \theta_{\mathrm{oa}}}{r},
$$

where $\sigma_{\mathrm{ow}}$ and $\sigma_{\mathrm{oa}}$ are respectively: the surface tension between oil and water and between oil and air. $\theta_{\text {ow }}$ and $\theta_{\text {oa }}$ are the wetting angle between the walls of the capillary as well as water and oil and oil and air.

The resistance to the flow of the viscous liquid in a straight cylindrical capillary in laminar conditions can be predicted using the equation of Hagen-Poiseuille. For the analyzed case in which two fluids flow in the cylindrical capillary, the pressure can be expressed as:

$$
p_{\mathrm{R}}=\frac{8 \eta_{\mathrm{w}} h_{\mathrm{w}} \mathrm{d} Q_{\mathrm{w}}}{\pi r^{4}}+\frac{8 \eta_{\mathrm{o}} h_{\mathrm{o}} \mathrm{d} Q_{\mathrm{w}}}{\pi r^{4}},
$$

where the first part of the equation refers to the height of water $h_{\mathrm{w}}$, while the second part refers the oil length $L_{\mathrm{o}}$ in the capillary. Both $h_{\mathrm{w}}$ and $L_{\mathrm{o}}$ are changing during flow. Using Eq. (2) it can be expressed as:

$$
h_{\mathrm{w}}=L-L_{\mathrm{o}}
$$

and after substituting to Eq. (5) we get:

$$
p_{\mathrm{R}}=\frac{8\left(\eta_{\mathrm{w}}\left(L-L_{\mathrm{o}}\right)+\eta_{\mathrm{o}} L_{\mathrm{o}}\right) \mathrm{d} Q_{\mathrm{w}}}{\pi r^{4}}
$$

The balance of forces governing the flow of liquid in the capillary tube can be expressed as:

$$
p_{\mathrm{R}}=p_{\mathrm{G}}+p_{\mathrm{C}}
$$

and after substituting equations (3), (4) and (7) in to the (8) one gets as follows:

$$
\frac{8\left(\eta_{\mathrm{w}}\left(L-L_{\mathrm{o}}\right)+\eta_{\mathrm{o}} L_{\mathrm{o}}\right) \mathrm{d} Q_{\mathrm{w}}}{\pi r^{4}}=\rho_{\mathrm{w}} g h+\rho_{\mathrm{o}} g L_{\mathrm{o}}+\frac{2 \sigma_{\mathrm{ow}} \cos \theta_{\mathrm{ow}}}{r}-\frac{2 \sigma_{\mathrm{oa}} \cos \theta_{\mathrm{oa}}}{r}
$$


After arranging the variables, Eq. (9) takes the form:

$$
\mathrm{d} Q_{\mathrm{w}}=\frac{\pi r^{4}}{8\left(\eta_{\mathrm{w}}\left(L-L_{\mathrm{o}}\right)+\eta_{\mathrm{o}} L_{\mathrm{o}}\right)}\left(\rho_{\mathrm{w}} g h+\rho_{\mathrm{o}} g L_{\mathrm{o}}+\frac{2 \sigma_{\mathrm{ow}} \cos \theta_{\mathrm{ow}}-2 \sigma_{\mathrm{oa}} \cos \theta_{\mathrm{oa}}}{r}\right)
$$

knowing that

$$
\mathrm{d} Q_{\mathrm{w}}=\frac{\mathrm{d} V}{\mathrm{~d} t},
$$

where $V$ is the volume of liquid leaving the capillary in time $t$ while the volume $V$ may be calculated using the formula:

$$
\mathrm{d} V=\pi r^{2} \mathrm{~d} L_{\mathrm{o}}
$$

Equation (10) can therefore be written as:

$$
\frac{\pi r^{2} \mathrm{~d} L_{\mathrm{o}}}{\mathrm{d} t}=\frac{\pi r^{4}}{8\left(\eta_{\mathrm{w}}\left(L-L_{\mathrm{o}}\right)+\eta_{\mathrm{o}} L_{\mathrm{o}}\right)}\left(\rho_{\mathrm{w}} g h+\rho_{\mathrm{o}} g L_{\mathrm{o}}+\frac{2 \sigma_{\mathrm{ow}} \cos \theta_{\mathrm{ow}}-2 \sigma_{\mathrm{oa}} \cos \theta_{\mathrm{oa}}}{r}\right)
$$

or after the arrangement of variables:

$$
\frac{\mathrm{d} L_{\mathrm{o}}}{\mathrm{d} t}=\frac{r^{2}}{8\left(\eta_{\mathrm{w}}\left(L-L_{\mathrm{o}}\right)+\eta_{\mathrm{o}} L_{\mathrm{o}}\right)}\left(\rho_{\mathrm{w}} g h+\rho_{\mathrm{o}} g L_{\mathrm{o}}+\frac{2 \sigma_{\mathrm{ow}} \cos \theta_{\mathrm{ow}}-2 \sigma_{\mathrm{oa}} \cos \theta_{\mathrm{oa}}}{r}\right)
$$

After distribution of variables, Eq. (14) takes the form:

$$
\mathrm{d} t=\frac{8\left(\eta_{\mathrm{w}}\left(L-L_{\mathrm{o}}\right)+\eta_{\mathrm{o}} L_{\mathrm{o}}\right)}{r^{2}\left(\rho_{\mathrm{w}} g h+\rho_{\mathrm{o}} g L_{\mathrm{o}}+\frac{2 \sigma_{\mathrm{ow}} \cos \theta_{\mathrm{ow}}-2 \sigma_{\mathrm{oa}} \cos \theta_{\mathrm{oa}}}{r}\right)} \mathrm{d} L_{\mathrm{o}}
$$

Equation (15) after integration enables to predict the height of both phases of the heights $h_{\mathrm{w}}$ and $L_{\mathrm{o}}$ at a given time.

Considering the really existing conditions, it is necessary to take into account the case when oil is completely removed from the capillary $L_{\mathrm{o}}=0$ and a single-phase flow only occurs. In this case, water in the capillary is washed out by water which is above the capillary. It can be written that the height of water in the capillary tube is $L_{\mathrm{w}}$ and changes with time.

This approach is analogous to the previous one for two-phase flow, however, it describes a case where only water flows in the capillary. It can be also expressed as:

$$
\begin{aligned}
h_{\mathrm{w}}+H & =h=\mathrm{const} \\
L_{\mathrm{w}}+h_{\mathrm{w}} & =L=\mathrm{const}
\end{aligned}
$$

In the case of a single-phase flow the gravitational pressure will be:

$$
p_{\mathrm{G}}=g \rho_{\mathrm{w}}\left(h+L_{\mathrm{w}}\right)
$$

The capillary pressure will be as follows, taking into account only the forces occurring at the water-air interface:

$$
p_{\mathrm{C}}=\frac{2 \sigma_{\mathrm{wa}} \cos \theta_{\mathrm{wa}}}{r}
$$

The flow resistance in this case is defined by the equation of Hagen-Poiseuille in the form of:

$$
p_{\mathrm{R}}=\frac{8 \eta_{\mathrm{w}} L}{\pi r^{4}} \mathrm{~d} Q_{\mathrm{w}}
$$

The pressure balance resulting from acting forces can be then expressed as follows:

$$
p_{\mathrm{R}}=p_{\mathrm{G}}+p_{\mathrm{C}}
$$


Fig. 2 Oil and water flow in the three capillaries of different radii

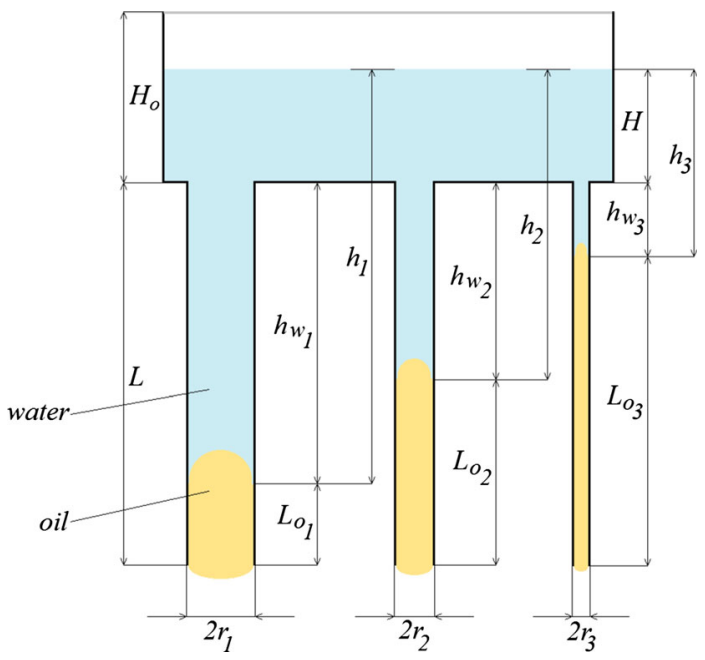

Substituting equations (18), (19) and (20) to the (21) the following formula can be derived:

$$
\frac{8 \eta_{\mathrm{w}} L}{\pi r^{4}} \mathrm{~d} Q_{\mathrm{w}}=g \rho_{\mathrm{w}}\left(h+L_{\mathrm{w}}\right)+\frac{2 \sigma_{\mathrm{wa}} \cos \theta_{\mathrm{wa}}}{r} .
$$

Knowing that

$$
\mathrm{d} Q_{\mathrm{w}}=\frac{\pi r^{2} \mathrm{~d} L_{\mathrm{w}}}{\mathrm{d} t}
$$

Equation (22) can be expressed as:

$$
\mathrm{d} t=\frac{8 \eta_{\mathrm{w}} L}{r^{2} \rho_{\mathrm{w}} g\left(h+L_{\mathrm{w}}\right)+2 \sigma_{\mathrm{wa}} \cos \theta_{\mathrm{wa}} r} \mathrm{~d} L_{\mathrm{w}}
$$

The results of the presented considerations provide a tool to predict the rate of removal of oily substances from the capillary as a function of time. It is also possible to determine the course of process of single-phase flow when the oil is completely removed.

\subsection{Gravitational Oil Elution from a Bundle of Capillaries}

The previous section included the analysis of the process of oil elution from a single capillary. A common approach in the literature (Carman 1937) is to present a porous structure of the medium as a bundle of parallel capillaries. The process of removing oily substances from such systems is complicated because the flow in one capillary will affect the flow in other capillaries. Such interactions may be explained using an example of three parallel capillaries of different radii $r_{1}, r_{2}, r_{3}$, and length $L$, connected with the bottom of the tank filled with the eluting liquid of height $H$, which at the beginning of the process is equal to $H_{0}$ and decreases with time, as shown in Fig. 2. Cross-sectional area of the tank is $A_{\mathrm{T}}$. In addition, at the beginning of the process capillaries are completely filled with a non-wetting liquid of the high viscosity-oil.

During the flow water will replace oil owing to action of gravity and capillary forces opposite to the flow resistance of viscous liquid. Due to different diameters, water level in the capillaries $h_{\mathrm{w}}$ will be different after a given time-Fig. 2 . As the result of the smallest flow resistance, it can be expected that the flow will be at the highest velocity in the capillary of 
the largest diameter and that in this capillary complete removal of oil be the earliest-water breakthrough. The phenomenon of a breakthrough will intensify the outflow of water from the tank and will accelerate the decrease of level $H$.

For the flow in a single capillary value $h$, defined by Eq. (1), has a constant value. In the case of the capillary bundle, the amount of water in the tank decreases due to the outflow from other capillaries. As a result of the interactions between capillaries $h$ values will vary during the flow. This case requires to use discretization approach, which implies a steady state of the system at small time intervals. Using this approach, it is possible to calculate the value of $L_{\mathrm{o}}$ or $L_{\mathrm{w}}$ based on Eqs. (15) and (24). Equations (2) and (17) allowed prediction of the amount of liquid which flowed to the capillary ducts in a given time period. On this basis it is possible to calculate the amount of liquid flowing out from the individual capillaries. By adding the volumes of liquid flowing out from all capillaries, we get the total volume of liquid $V_{\text {out }}$ which was removed from the bed. Knowing this value we can calculate the liquid volume change in the tank and determine the change of the height of the liquid above the capillaries according to the equation:

$$
H=\frac{V_{i}-V_{\text {out }}}{A_{\mathrm{T}}},
$$

where $V_{i}$ is the initial volume of liquid in the tank, $A_{\mathrm{T}}$ is the cross-sectional area of the tank.

Such approach allows to relate the presented model to the real system.

Using Eq. (25), the height $H$ is taken as an initial value for the calculations in subsequent periods of time. This cycle is repeated until $H=0$. Based on these calculations, it is possible to determine how the flow rate of fluid varies with time and what volume of oil flows out from a bundle of capillaries of different diameters.

\subsection{Conversion of Porous Bed to a Bundle of Capillaries}

The application of the above considerations for the development of a capillary model of elution of highly viscous substances from the inside of porous structures required the development of relationships by means of which it was possible to replace the granular bed with a bundle of capillaries of specific diameters and lengths. For this purpose there was proposed an approach, based on the similarity of grains size distribution to the distribution of the diameter size of capillaries. This assumption allows for the calculation of diameters and the number of capillaries in accordance with the following procedure.

Knowing that the volume of granular bed is equal to $V_{\mathrm{B}}$, where the volume of free space is $V_{\mathrm{F}}$, the porosity of the bed $\varepsilon$ can be expressed as:

$$
\varepsilon=\frac{V_{\mathrm{F}}}{V_{\mathrm{B}}}
$$

Knowing the distribution of grain diameters in the bed, on the basis of the sieve analysis, it is possible, after making the assumption of proportionality, to determine the distribution of capillaries diameters. The radius of capillaries for each class of grain size distribution can be calculated from the Carman (1937) relationship:

$$
r_{j}=\frac{1}{6} d_{\mathrm{gr}_{j}}\left(\frac{\varepsilon}{1-\varepsilon}\right),
$$

where $d_{\mathrm{gr}_{j}}$ is the average grain diameter for the analyzed class. 
Capillary height $L$ can be expressed as a product of the capillary tortuosity $K_{\mathrm{t}}$ and bed height $L_{\mathrm{B}}$ :

$$
L=K_{\mathrm{t}} L_{\mathrm{B}}
$$

Knowing the radius $r_{j}$ and height $L$ of the capillary, it is possible to calculate the volume of the capillary $V_{\text {cap }}$ from the formula:

$$
V_{\text {cap }_{j}}=\left(\pi r_{j}^{2}\right) L
$$

Assuming that the share $\varphi_{j}$ of individual classes of grain diameters is proportional to the corresponding classes of capillaries, we can calculate what volume of free space will be occupied by capillaries of given radii $V_{\mathrm{fc}_{j}}$

$$
V_{\mathrm{fc}_{j}}=V_{\mathrm{B}} \varphi_{j}
$$

On this basis it is possible to determine the number of capillaries $n_{j}$ in a given class according to the relationship:

$$
n_{j}=\frac{V_{\mathrm{fc}_{j}}}{V_{\mathrm{cap}_{j}}}
$$

Adopting the above assumptions, the total volume of all the capillaries corresponds to the void space of the bed.

Based on the above assumptions, it is possible to convert the granular bed layer of a given height $L_{\mathrm{B}}$ and diameter $D_{\mathrm{B}}$ to a bundle of capillaries of different diameters.

\subsection{The Preferential Flow Paths}

The description of the process of oil elution from the granular bed by means of a capillary bundle model does not take into account for certain phenomena occurring in the bed, related to "viscous fingering" phenomenon described by Homsy (1987), which generally refers to the onset and evolution of instabilities that occur in the displacement of fluids in porous materials. Therefore, it is necessary to adopt additional assumptions that allow for better representation of the flow conditions in the actual granular bed.

In a porous bed liquid flows through the paths of various cross sections and shapes. These paths are interconnected and form a network. In the situation when the flow driving force resulting from gravity and capillary pressure is small, the liquid flowing in a path with a small cross-section may enter the path where the flow resistance is smaller. Therefore, the flow in a path of small cross-section is stopped and the liquid flows through preferential paths. The model of capillary bundle assumes flow of liquid along the capillaries but it does not include the connection between these capillaries. Therefore, to take into account the effect of passing of the liquid from the smaller capillaries in the situation when the driving force is insufficient to overcome prevailing resistance force, there was introduced the concept of a minimum flow pressure $p_{\mathrm{m}}$. This value refers to the such a small pressure at which the flow in the capillary is stopped. The liquid, which would flow through this capillary, will be forced to flow through the capillaries of larger diameters. As the result of the introduction of this value it is possible to represent the phenomenon of redirection of flow between the paths in the model of capillaries network.

Therefore one can assume that the minimum pressure of the flow is inversely proportional to the diameter of the capillary, and can be expressed by the formula:

$$
p_{\mathrm{m}_{j}}=\frac{\zeta}{r_{j}}
$$


where $\zeta$ is the coefficient of proportionality.

\subsection{Programming}

Let the granular layer of height $L_{\mathrm{B}}$, cross-sectional diameter $D_{\mathrm{B}}$ and the porosity $\varepsilon$ be replaced by a bundle $N$ of capillaries of height $L$. Among the capillaries there is a number of $j$ different groups of a given value of radius $r_{j}$. The number of capillaries in a group is $n_{j}$. All capillaries are initially fully saturated with non-wetting liquid (oil) and above them there is wetting liquid column (water) of height $H$ and volume $V_{i}$. The flow of liquid in such bundle of capillaries will take place according to the same laws as during the elution of oil from the three capillaries of different diameters. A numerical program can be developed to calculate the amount of oil flowing out from the bed and the change of liquid flow velocity over time. To illustrate the operation of this program and the method of calculating the individual values based on all the above assumptions, a block diagram is shown in Fig. 3.

This program assumes the following conditions:

$$
\begin{aligned}
& \text { for } t=0 \quad H=H_{0} \\
& \text { for } t=t_{\text {end }} \quad H=0
\end{aligned}
$$

where $H_{0}$ is the initial height of water column above the capillaries, $t_{\mathrm{end}}$ is the final time of the process.

At the beginning of the process the height of oil present in the capillary is equal to the capillary height $L_{\mathrm{O}_{j}}=L$ while the amount of water percolated to the capillary is equal to zero $h_{\mathrm{w}_{j}}=0$.

To start the calculations, it is necessary to determine pressure $p$ over the inlets to capillaries, in order to compare it with the value of the minimum pressure $p_{\mathrm{m}_{j}}$. Water column pressure at the inlet to the capillary can be expressed by the relationship:

$$
p=\rho_{\mathrm{w}} g H
$$

If value $p$ is smaller than the value $p_{\mathrm{m}_{j}}$ then in the capillaries of given sizes the flow of fluid will not occur, $h_{\mathrm{w}_{j}}=0$. However, if the value $p$ is greater than value $p_{\mathrm{m}_{j}}$ flow will take place.

Depending on whether in a given group a breakthrough of water took place $\left(h_{\mathrm{w}_{j}}>L\right)$ or not $\left(h_{\mathrm{w}_{j}} \leq L\right)$, the next value of the height of liquid flowing at a given time in a group of capillaries will be calculated from two different equations. For the case when oil is still present in the capillary, when water breakthrough has not yet taken place, flow will be described on the basis of Eq. (15), which will be integrated in the range:

$$
\int_{t_{i}}^{t_{i+1}} \mathrm{~d} t=\alpha \int_{L_{\mathrm{o}_{i}}}^{L_{\mathrm{o}_{i+1}}} \frac{8\left(\eta_{\mathrm{w}}\left(L-L_{\mathrm{o}}\right)+\eta_{\mathrm{o}} L_{\mathrm{o}}\right)}{r^{2}\left(\rho_{\mathrm{w}} g h+\rho_{\mathrm{o}} g L_{\mathrm{o}}+\frac{2 \sigma_{\mathrm{ow}} \cos \theta_{\mathrm{ow}}-2 \sigma_{\mathrm{oa}} \cos \theta_{\mathrm{oa}}}{r}\right)} \mathrm{d} L_{\mathrm{o}},
$$

where $\alpha$ is the parameter of the equation.

After water breakthrough $\left(h_{\mathrm{w}_{j}}>L\right)$, the flow will be described according to Eq. (24), which will be integrated in the range:

$$
\int_{t_{i}}^{t_{i+1}} \mathrm{~d} t=\beta \int_{L_{\mathrm{w}_{i}}}^{L_{\mathrm{w}_{i+1}}} \frac{8 \eta_{\mathrm{w}} L}{r^{2} \rho_{\mathrm{w}} g\left(h+L_{\mathrm{w}}\right)+2 \sigma_{\mathrm{wa}} \cos \theta_{\mathrm{wa}} r} \mathrm{~d} L_{\mathrm{w}},
$$

where $\beta$ is the parameter of the equation. 


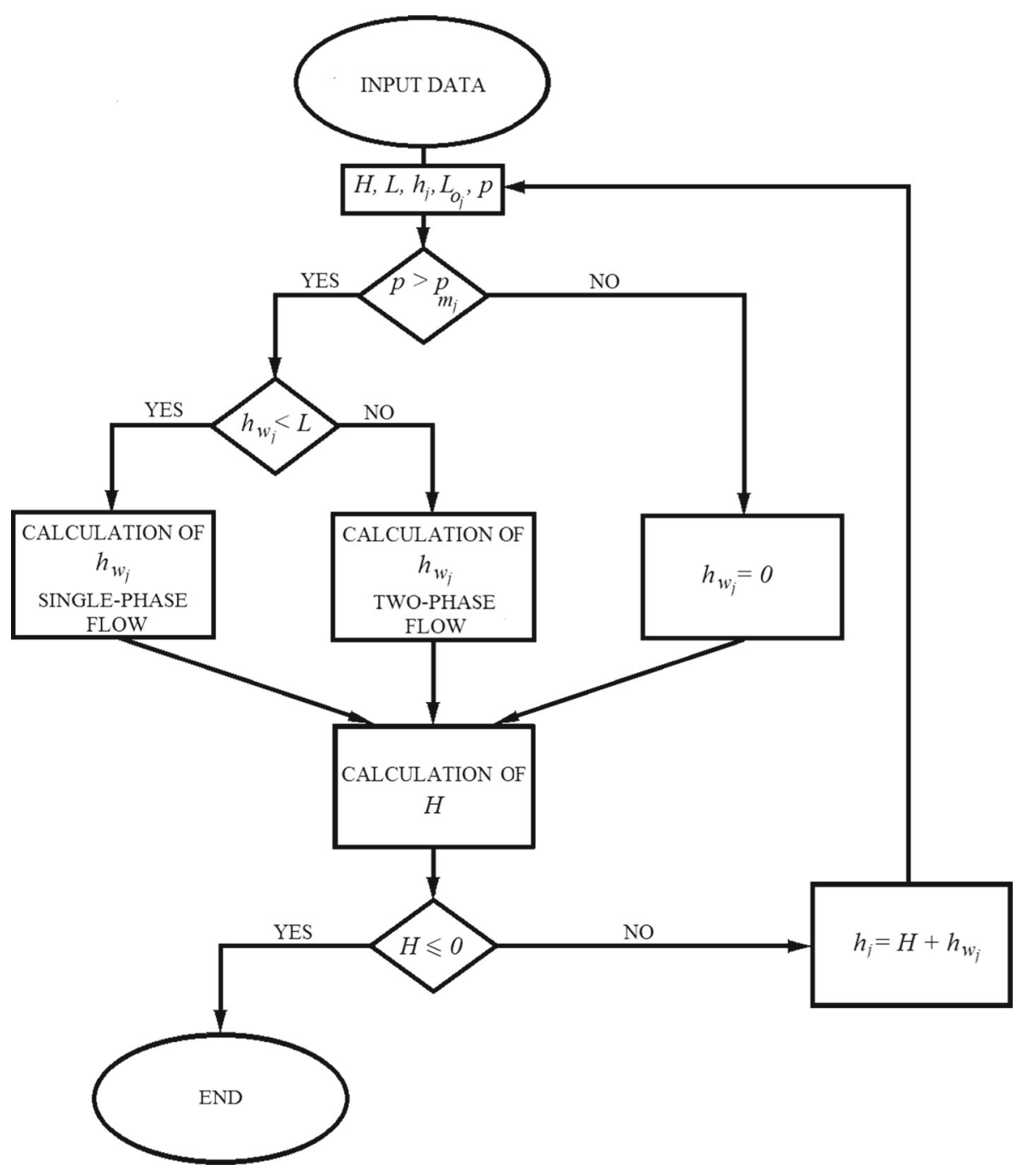

Fig. 3 Block diagram of the program

Knowing the changes of liquid height $h_{\mathrm{w}_{j}}$ in individual groups of capillaries, it is possible to calculate the total volume of liquid $V_{\text {out }}$ that flowed from capillaries at that time, which makes it possible to calculate a new height of the liquid column over the capillaries by means of Eq. (25). If the received height $H$ is greater than zero, then a new length of water column $h_{j}$ is calculated from the following relationship:

$$
h_{j}=H+h_{\mathrm{w}_{j}}
$$

When the liquid height above capillaries $H$ becomes equal to zero, the process is stopped.

Knowing the changes of volumes of oil $V_{\text {out }}$, which outflows from the bed during the elution process, it is possible to calculate the changes of bed saturation $S_{\mathrm{O}}$ in time on the basis of equation: 


$$
S_{\mathrm{o}}=\frac{V_{\mathrm{F}}-V_{\text {out }}}{V_{\mathrm{F}}}
$$

\subsection{Taking into Consideration the Incomplete Saturation of the Bed with Oil}

The above considerations were related to the cases where the granular bed was completely initially saturated with oily substance, $S_{\text {io }}=1$. In real cases we rarely deal with such situation, when all free spaces of the bed are filled with oil. Often there is a situation of an incomplete saturation, where only a part of the pores is filled.

Taking into consideration an incomplete saturation of the bed one should to assume that at the beginning of the process, oil is present only in a part of the capillaries and the rest of them is empty. This means that two-phase flow will take place only in a part of the capillaries, while in the remaining part only a single-phase flow will take place. The share of capillaries, in which elution takes place, results from the initial saturation of bed with oil $S_{\text {io }}$. Thus, the number of capillaries in a given size class $n_{\mathrm{o}_{j}}$ in which oil flow occurs can be calculated from the relationship:

$$
n_{\mathrm{o}_{j}}=S_{\mathrm{io}} n_{j}
$$

While the number of capillaries in which only water flow occurs $n_{\mathrm{w}_{j}}$ can be expressed as:

$$
n_{\mathrm{w}_{j}}=\left(1-S_{\mathrm{io}}\right) n_{j}
$$

For the capillaries filled with oil, flow will take place according to the diagram presented on the Fig. 3, while for empty capillaries only water will flow (relationship 36). This approach enables to calculate the oil elution only from a part of capillaries and to take into consideration changes of the level of washing liquid caused by the outflow of water from the capillaries which are not filled with oil.

On the basis of model calculations is possible to determine the change in liquid height above the bed and the amount of oil which flows out of the bed during the process of elution.

\section{Experimental}

The testing equipment included an experimental column made of vertical glass tube of internal diameter $0.05 \mathrm{~m}$ and height $0.7 \mathrm{~m}$, mounted on a tripod. Granular material layer, placed in it, was secured at the bottom with a strainer to prevent the falling of bed from the column. Granular material saturated with oil was placed in the column to the defined height. Waterwashing liquid-was passed through the bed, at the beginning of the process the height of water above the bed amounted $0.35 \mathrm{~m}$. The investigated process could be followed owing to the scale mounted on the column and by means of a stopwatch which helped to determine the fall time of liquid column relative to the granular layer every $0.01 \mathrm{~m}$. The flowing out liquid was collected in beakers in successive time intervals corresponding to the falling by $0.05 \mathrm{~m}$ of the liquid column above the bed and was tested for determining the concentration of the flowing out oil. The methodology for determining the concentration of oil in the subsequent portions of the liquid flowing out from the bed was based on turbidimetric tests, which were presented in the works of Lemarchand et al. (2003) and Sęk et al. (2011).

During the tests, vegetable oil "Bartek" ZT Kruszwica SA was used as highly viscous substance eluted from the bed and washing liquid was water. The measurements were carried out in an ambient temperature. The granular bed consisted of glass microspheres of different grain fractions. For each bed an analysis of particle size distribution was made, using microscopic image analysis. 
During the tests there were made measurements of high viscosity substance elution from the granular bed with a liquid of lower viscosity at different grain diameters, various bed heights and various degrees of bed saturation with oil.

In the case at study of the influence of the various bad grains diameter there were used beds of glass spheres with a particle size fraction of 200-300, 300-400, 400-600 $\mu \mathrm{m}$ of porosity respectively $0.34,0.36,0.36$. The height of the bed in the column in every measurement was equal to $0.2 \mathrm{~m}$, the bed saturation with oil was equal to $S_{\text {io }}=1$.

During the study of the influence of the height of the porous medium on the elution process the bed of grain-size fraction of 200-300 $\mu \mathrm{m}$ was used; it was placed in the experimental column to the height of $0.1,0.15,0.2,0.25,0.3 \mathrm{~m}$. The degree of initial saturation of the bed here was also $S_{\text {io }}=1$.

In the course of the study of the influence of the degree of saturation was used a granular bed of particle size fraction 200-300 $\mu \mathrm{m}$ and height $0.2 \mathrm{~m}$. The bed was mixed with oil in such amounts that it corresponded to the degrees of saturation $S_{\mathrm{io}}: 53,71,88$ and $100 \%$.

\section{Application of the Capillary Model}

The application of capillary model of elution of high viscosity substance from the inside of the porous medium due to gravitational forces, presented in Sect. 2, required the sieve analysis of the granulated material. Based on these measurements, the total width of distribution of diameters was determined as well as the range of diameters of grains in every distinguished class $z_{\mathrm{d}}$.

Then the average grain diameter $d_{\mathrm{gr}_{j}}$, which was used in Eq. (27), calculated for the analyzed class of capillaries $z_{\mathrm{p}_{j}}$ can be determined on the basis of the equation:

$$
d_{\mathrm{gr}_{j}}=z_{\mathrm{p} \min _{j}}+\frac{z_{\mathrm{p} \max _{j}}-z_{\mathrm{p} \min _{j}}}{2}
$$

where $z_{\mathrm{p}} \min _{j}$ value of the minimum diameter of the grains in the class $z_{\mathrm{p} \text { max }}$ the maximal value of the diameter of the grains in the class.

The diameters of the capillaries corresponding to the diameters of grains $d_{\mathrm{gr}}$ in the given class were assumed to be proportional with $\xi$ as the coefficient of proportionality. For the analyzed beds proportionality coefficient $\xi$ equal to the value of 5 was assumed. Proceeding according to the course of the calculations presented in Sect. 2.3, it was possible to calculate the capillary radii $r_{j}$ and their numbers $n_{j}$ for the given classes of capillary sizes in a particular bed.

Knowing also all the necessary physico-chemical properties of the eluted oil-viscosity $\eta_{\mathrm{o}}=60 \mathrm{mPas}$, density $\rho_{\mathrm{o}}=885 \mathrm{~kg} / \mathrm{m}^{3}$, interfacial tension $\sigma_{\mathrm{ow}}=0.048 \mathrm{~N} / \mathrm{m}$ and $\sigma_{\mathrm{oa}}=0.024 \mathrm{~N} / \mathrm{m}$, wetting angles: $\theta_{\mathrm{ow}}=30^{\circ}, \theta_{\mathrm{oa}}=0^{\circ}$; washing water-viscosity $\eta_{\mathrm{w}}=0.99 \mathrm{mPas}$, density $\rho_{\mathrm{w}}=997 \mathrm{~kg} / \mathrm{m}^{3}$ and interfacial tension $\sigma_{\mathrm{wa}} 0.072 \mathrm{~N} / \mathrm{m}$, wetting angle $\theta_{\text {wa }}=0^{\circ}$; parameters of the bed-diameter $D_{\mathrm{B}}=0.05 \mathrm{~m}$, length $L_{\mathrm{B}}$, porosity $\varepsilon$, tortuosity $K_{\mathrm{t}}=1.44$ (Carman 1937) and the initial height $H_{0}$ of the water column above the bed it was possible to apply the presented capillary model. Based on the assumptions shown in the block diagram presented in Fig. 3, a computer program was developed what allowed to determine changes of the height level in the liquid column above the bed of $H$ and the level of bed saturation with oil $S_{\mathrm{o}}$ over time. 
(a)

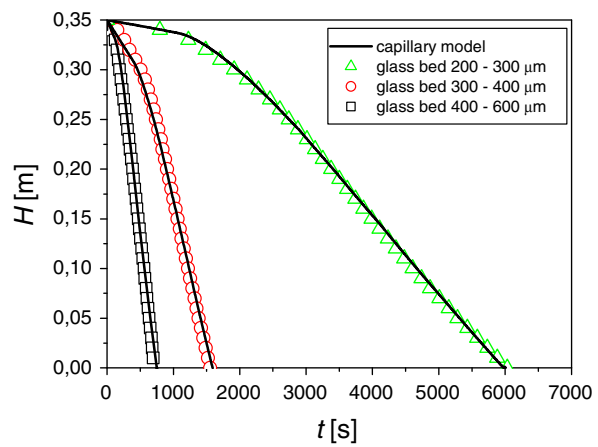

(b)

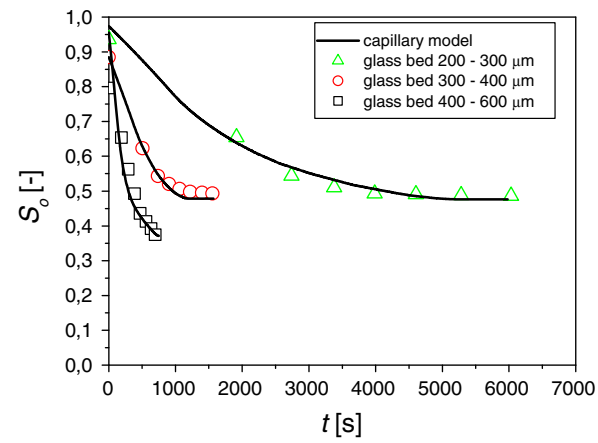

Fig. 4 Relationship a $H(t)$ and $\mathbf{b} S_{\mathrm{o}}(t)$ for different beds for the experimental data and the results of model calculations

Table 1 Parameters of a capillary model depending on the type of bed

\begin{tabular}{llll}
\hline Glass beads $(\mu \mathrm{m})$ & Parameter $\alpha$ & Parameter $\beta$ & Parameter $\zeta$ \\
\hline $200-300$ & 0.41 & 4.51 & 0.25 \\
$300-400$ & 0.32 & 2.03 & 0.75 \\
$500-600$ & 0.15 & 1.52 & 1.21 \\
\hline
\end{tabular}

\subsection{The Influence of the Granulation of the Bed}

On the basis of experimental studies of the process of gravitational elution of a high viscosity substance from the granular bed by means of a liquid of lower viscosity it was possible to compare the results of model calculations with the experimental data.

The changes in the height of the liquid column above the bed (Fig. 4a) and changes in the degree of saturation of bed with oil (Fig. 4b) during the elution process for the experimental data and the results of model calculations for different granular beds has been shown. As can be seen, for medium with smaller grain sizes elution process time is longer. The amount of oil eluted from the bed decreases with time and eventually reaches a constant value. For beds with larger particle sizes a greater degree of total elution has been achieved. Generally one can find that in every case about $30-40 \%$ of oil will remain in the bed independently on the grains sizes.

The results of model calculations developed on the basis of the assumptions set out in Sect. 2 give a good matching for all analyzed beds of glass beads. The model included three parameters: $\alpha, \beta$ and $\zeta$, which are presented in Table 1 . The parameter $\alpha$ refers to two-phase flow (Eq. 35) and determines the oil flow rate in the capillary before the occurrence of the water breakthrough. The parameter $\beta$ refers to a single-phase flow (Eq. 36) when there is only water in the capillaries. For beds with larger grain size parameters $\alpha$ and $\beta$ take smaller values, while the parameter values increase with increasing grain size.

\subsection{The Influence of the Bed Height}

The results of model calculations made on the basis of presented assumptions were compared with the experimental data for the process of oil substances elution from beds of different heights, which is presented in Fig. 5a, b. As can be seen, an increase in the height of the bed caused an increase in duration of the elution process and a reduction of degree of elution. 
(a)

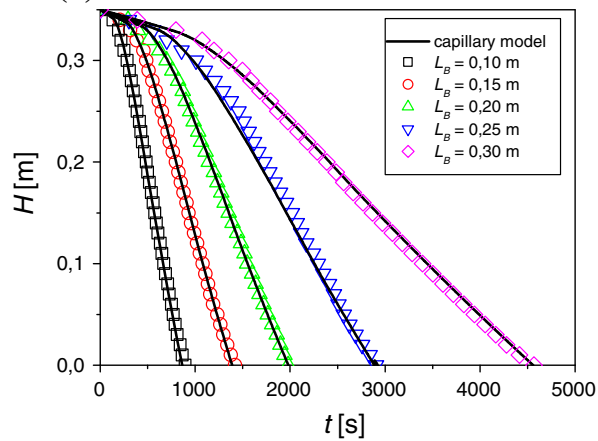

(b)

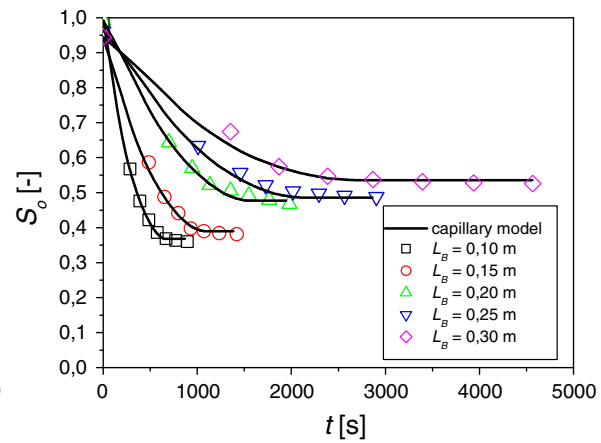

Fig. 5 Relationship a $H(t)$ and $\mathbf{b} S_{\mathrm{O}}(t)$ at different bed lengths for the experimental data and the results of model calculations

Table 2 Parameters of capillary model depending on the bed height

\begin{tabular}{llll}
\hline Bed height $(\mathrm{m})$ & Parameter $\alpha$ & Parameter $\beta$ & Parameter $\zeta$ \\
\hline 0.10 & 0.32 & 1.61 & 0.67 \\
0.15 & 0.32 & 1.75 & 0.71 \\
0.20 & 0.32 & 2.01 & 0.75 \\
0.25 & 0.32 & 2.82 & 1.03 \\
0.30 & 0.32 & 4.52 & 1.41 \\
\hline
\end{tabular}

One can observe that when the beds have lower heights the oil elution is greater than in the case of higher beds.

On the basis of model calculations based on the assumptions presented in Sect. 2 it was possible to examine how the presented model allows to predict the influence of the bed height. In each analyzed case a good compatibility with the experimental data was obtained. The summary of model parameters is presented in Table 2. Parameter $\alpha$ was independent of the length of the bed, while the dependence of values of the parameters $\beta$ and $\zeta$ was found to be growing with the height of the bed.

\subsection{The Influence of the Initial Saturation of the Bed with Oil}

Taking into account the results of experiments of the elution of high viscosity substances from the granular bed at different initial degree of saturation, it was possible to verify the suitability of this capillary model, which is shown in Fig. 6a, b. The duration of elution process was decreasing with drop in the initial saturation of bed with oil. It was important that the final elution ratio of oil from the bed reached the same value at about $50 \%$, regardless of the initial degree of saturation.

The calculation results obtained on the basis of the model presented in Sect. 2 give a good matching for both the liquid column height changes and the changes in the bed saturation level with oil over time for each analyzed initial saturation. The values of parameters $\alpha, \beta$ and $\zeta$ are presented in Table 3. Parameters $\alpha$, and $\beta$ show decreasing trends along with the decrease of the initial bed saturation with oil. However, parameter $\zeta$ increases with decreasing value $S_{\text {io }}$. 


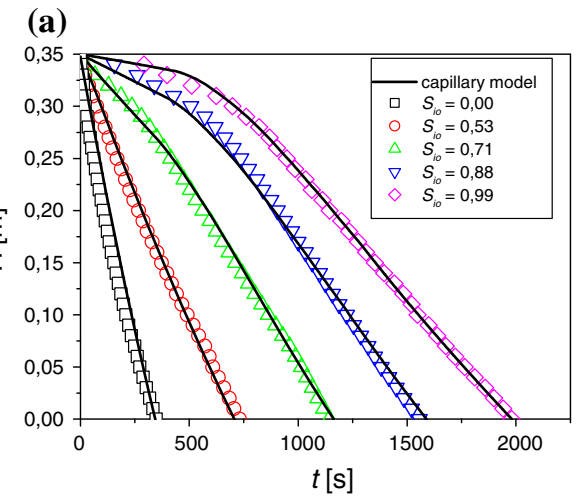

(b)

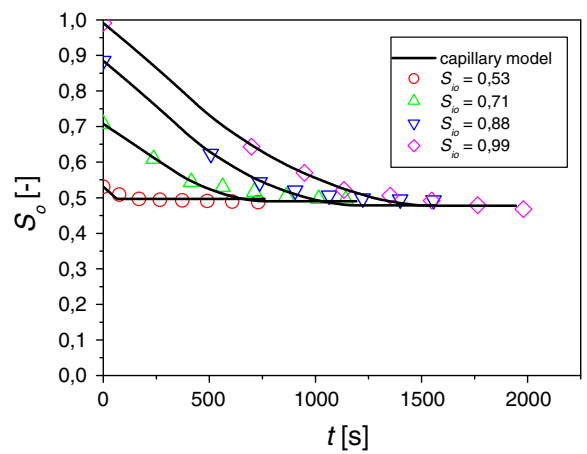

Fig. 6 Relationship a $H(t)$ and $\mathbf{b} S_{\mathrm{O}}(t)$ for the experimental data and the results of model calculations. Bed $300-400 \mu \mathrm{m}$

Table 3 Parameters of capillary model depending on the degree of bed saturation

\begin{tabular}{llll}
\hline Saturation $S_{\mathrm{io}}(-)$ & Parameter $\alpha$ & Parameter $\beta$ & Parameter $\zeta$ \\
\hline 0.97 & 0.400 & 4.5 & 0.25 \\
0.75 & 0.250 & 2.4 & 1.10 \\
0.56 & 0.100 & 1.9 & 2.20 \\
0.37 & 0.030 & 0.9 & 3.00 \\
0.00 & 0.002 & 0.5 & - \\
\hline
\end{tabular}

\section{Discussion on the Modeling Predictions}

In order to determine how the model predicts the dependence of the individual parameters characterizing flowing liquids and the bed on the process of elution a discussion was held on the impact of viscosity. $\eta_{\mathrm{o}}$ of eluted liquid (oil), viscosity. $\eta_{\mathrm{w}}$ of washing liquid (water) and the height of the washing liquid column $H_{\mathrm{o}}$ on the speed of the process and the amount of eluted oily substance. For this purpose model calculations were performed for elution of oil from the bed of glass beads with a grain size from 300 to $400 \mu \mathrm{m}$ and porosity $\varepsilon=0.36$ with a height $L_{\mathrm{B}}=0.2 \mathrm{~m}$. The values of the adopted parameters $\alpha, \beta$ and $\zeta$ amounted respectively: $0.32,2.03$ and 0.75 as in the Table 1 .

\subsection{Influence of the Viscosity of the Eluted Liquid}

Assuming different values of oil viscosity, with other parameters unchanged, it was possible to draw dependence graphs of water level changes above the bed (Fig. 7a) and changes in the degree of oil saturation (Fig. 7b) during the process.

According to the model assumptions the increase of the viscosity of the eluted liquid results in the increase of elution time as well as the increase of the degree of oil elution from the bed. Higher viscosity of oil substance results in a slower elution, especially in the first stage of the process, when the water has not yet made the flow paths. This means an increase in liquid flow resistance during two-phase transport. At high oil viscosity values, the final degree of the bed saturation.is bigger than in the cases of low viscosity, when the residual saturation is low, see Fig. 7c. The results of model calculations are consistent with the physical phenomena observed in reality (Kajdas 1979). The calculated degree of elution 
(a)

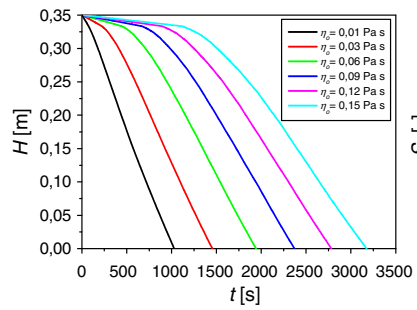

(b)

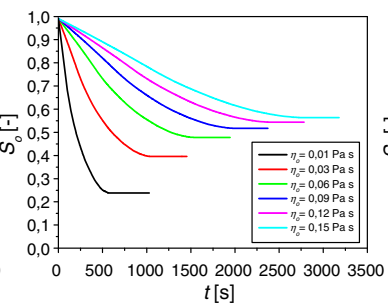

(c)

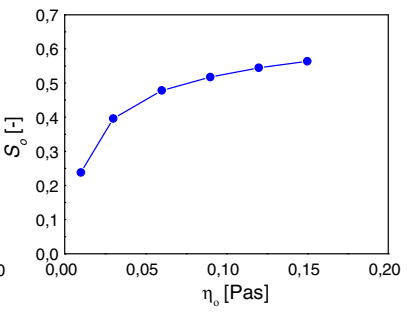

Fig. 7 Relationship a $H(t)$, b $S_{0}(t)$ and $\mathbf{c} S_{0}\left(\eta_{\mathrm{o}}\right)$ for different viscosity values of the eluted liquid calculated basing on modeling

(a)

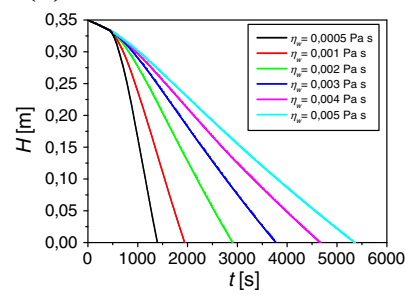

(b)

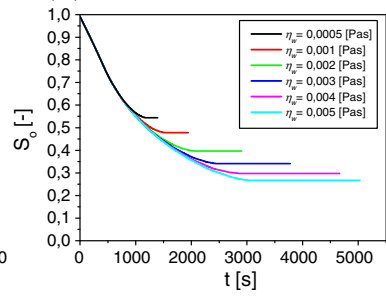

(c)

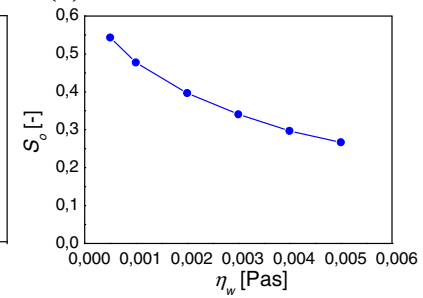

Fig. 8 Relationship a $H(t), \mathbf{b} S_{\mathrm{o}}(t)$ and $\mathbf{c} S_{\mathrm{o}}\left(\eta_{\mathrm{w}}\right)$ for different viscosity values of the washing liquid calculated on the basis of modeling

is the highest at the lowest values of viscosity of oil. It decreases with an increase of oil viscosity, however the changes are gradually smaller.

\subsection{Influence of the Washing Liquid Viscosity}

In order to verify how the presented model determines the influence of the viscosity of washing liquid on the elution process of oily substance from the inside of the porous structure, there were drawn graphs of changes of the level of the liquid column above the bed (Fig. 8a) and changes of the saturation level of the oily substance (Fig. 8b) over time for the results obtained from model calculations at different viscosity values $\eta_{\mathrm{w}}$.

The increase of washing liquid viscosity causes a significant increase of the time of falling of liquid column above the bed, especially in the final stages of the elution process i.e., when in most capillaries water breakthrough has occurred. Increased washing liquid viscosity causes greater flow resistance, which significantly slows down the process. However, the increase of this value also increases the degree of oil washing from the bed. The amount of residual oil remaining in the bed after the elution process is smaller when using a higher viscosity liquid. This means that despite a significant slowing down of elution process, it is possible to achieve greater efficiency of elution, see Fig. 8c. It should be noted that the increase in viscosity of the washing liquid causes more and more smaller increase in the degree of elution. This means that the continuous increase of the washing liquid viscosity causes less and less impact on the degree of oil removal from the bed. This is due to the fact that higher viscosity liquid cannot reach small pores and wash oil from them.

These results confirm the phenomena described in the literature (Bedient et al. 1994; Brooks and Cory 1966; Kajdas 1979) where the elution of the oily substance is dependent on 
(a)

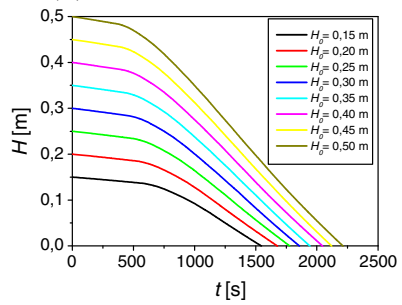

(b)

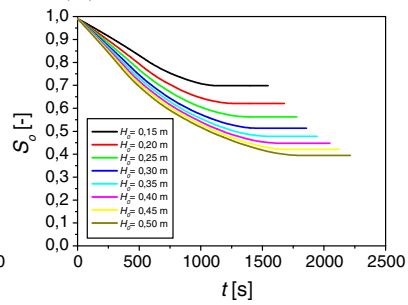

(c)

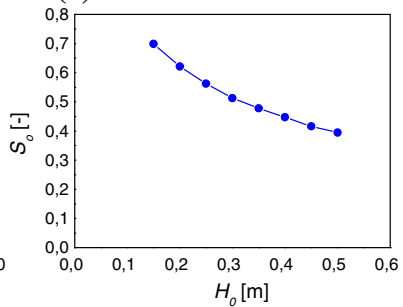

Fig. 9 Relationship a $H(t)$, b $S_{\mathrm{O}}(t)$ and $\mathbf{c} S_{\mathrm{o}}\left(H_{0}\right)$ for different initial values of liquid column height above the bed calculated on the basis of modeling

the ratio of the viscosity of the eluted liquid to the washing liquid. The higher the values of the ratio, the higher is the elution effectiveness. However, at high values of this ratio, further increasing of the washing liquid viscosity results in slight changes of the degree of oily liquid elution.

\subsection{Influence of the Height of the Washing Liquid Column Above the Bed}

An important parameter in the described case of elution of oily substance is the amount of washing substance used in the process. To see how changes of this parameter are predicted by the present model there are shown dependency graphs of changes of the height of liquid column (Fig. 9a) above the bed and the changes of bed saturation level with oil (Fig. 9b) over time at different initial values of the liquid column height above the bed $H_{0}$.

The increase of the washing liquid column shortens the first stage of elution i.e., acceleration of water breaking through the oily bed. Due to the greater amount of liquid that flows through the bed we can also observe a slight increase in the flow time through the bed. In the case of higher initial values of the water column the oil elution ratio is higher, however, the increase is not proportional as in is shown in Fig. 9c. This means that the use of significant amounts of water in the process of gravitational elution causes a slight increase in efficiency of the removal of highly viscous liquid from the granular bed.

\section{Conclusions}

On the basis of the performed research work it was found that it was possible to develop models of the elution process of highly viscous substances with a liquid of lower viscosity from a porous medium, based on the concept of bundles of capillaries representing granular bed of specified dimensions. This approach enabled to describe phenomena occurring during the flow taking place under the influence of gravitational forces. In result of the works on the model it was possible to predict the changes in time of such values as: relative permeability of the bed, degree of bed saturation with eluted substance, liquid flow rates, flow resistance, volumes of washing liquid.

In relation to the existing literature the following new physical phenomena and parameters characterizing the process were taken into account and can be regarded as achievement of the work.

Model assumptions allowed for conversion of the granular bed of defined dimensions with a given grains diameter distribution into the structures of the bundle of capillaries 
of corresponding distribution of their diameters. Such approach for the cases of modeling of elution processes was not considered. Models, existing in the literature, only allow the tracking of flow in capillary bundle, but do not refer to the actual composition of the granular bed.

The literature review reveal the capillary models related to the elution processes where in each capillary oil elution is present, while they not analyze the case, what happens when the oil is eluted from part of the capillaries. Assumptions used in this work allow to track elution process of high viscosity liquid by a lower viscosity fluid in the bundle of capillaries, taking into account fact that in the capillaries of a larger diameter water breakthrough phenomena occur more rapidly, while in the smaller capillaries this phenomenon occurs later. This means that in part of capillaries the aqueous phase only flows, while at the same time in remaining capillaries there is still a two-phase flow.

Modeling allowed to take into account non steady flow conditions with the driving force decreasing with time. This makes it possible to predict the behavior of the highly viscous substances in porous media such as soil structures under the influence of precipitation. In the majority of already developed capillary models driving force is constant, which significantly reduces their use. Approach adopted here allows the use of these models for specific cases of flow observed in reality.

During the elution process, phenomenon of fluid flow in the granular bed through the preferential paths, where the flow resistance is smallest, occurs. Capillary models described in the literature, ignore this phenomenon, assuming that the flow will take place through the whole bundle of capillaries. In the presented model this phenomenon has been included and dependent on the changing in time driving force. This concept enables the determination of residual oil bed saturation after the elution process. Developed model allows also to predict the final stages of elution as the function of the oil viscosity or as the dependence of the viscosity of washing liquid. Also the influence of the height of the washing liquid layer can be here studied.

Developed capillary models can become much more effective, than currently used in practice, tool to estimate the spread of oil pollution in the soil structure and to estimate the degree of crude oil extraction from porous media.

Acknowledgments The authors acknowledge the financial support provided by National Science Center through the Preludium program, Project number: 2013/05/N/ST8/03798.

Open Access This article is distributed under the terms of the Creative Commons Attribution License which permits any use, distribution, and reproduction in any medium, provided the original author(s) and the source are credited.

\section{References}

Ahmadlouydarab, M., Liu, Z.S., Feng, J.J.: Interfacial flows in corrugated microchannels: flow regims, transitions and hysteresis. Int. J. Multiph. Flow 37, 1266-1276 (2011)

Aranyosi, A.J.: Calculating the Velocity of Gravity- and Capillarity-Driven Flow. The Micromechanics Group, Research Lab of Electronics, MIT (2010). http://umech.mit.edu/HST410/flowvelocity.pdf

Bedient, P.B., Rifai, H.S., Newell, C.J.: Ground Water Contamination: Transport and Remediation. PTR Prentice Hall, Englewood Cliffs (1994)

Brooks, R.H., Cory, A.T.: Properties of porous media affecting fluid flow. J. Irrig. Drain. Div. Proc. ASCE 92(2), 61-88 (1966)

Carman, P.C.: Fluid flow through granular beds. Trans IChemE 15, 150-166 (1937)

Dahle, H.K., Celia, M.A., Hassanizadeh, S.M.: Bundle-of-tubes model for calculating dynamic effects in the capillary-pressure-saturation relationship. Transp. Porous Media 58, 5-22 (2005) 
Dullien, F.A.L.: Porous Media Fluid Transport and Pore Structure. Academic Press, San Diego (1992)

Fulcher, R.A., Ertekin, T., Stahl, C.D.: Effect of capillary number and its constituents on two-phase relative permeability curves. J. Pet. Technol. 2, 249-260 (1985)

Govindarajan, R., Sahu, K.C.: Instabilities in viscosity-stratified flow. Annu. Rev. Fluid Mech. 46, 331-353 (2014)

Homsy, G.M.: Viscous fingering in porous media. Annu. Rev. Fluid Mech. 19, 271-311 (1987)

Idorenyin, E., Shirif, E.: A new simulation model for two-phase flow in porous media. Braz. J. Pet. Gas 6(1), 001-018 (2012)

Kajdas, C.: Chemistry and Physical Chemistry of Petroleum. Scientific and Technical Publisher, Warsaw (1979) (in Polish)

Kembłowski, Z., Michałowski, S., Strumiłło, C., Zarzycki, R.: Theoretical Basis of Chemical and Process Engineering. Scientific and Technical Publisher, Warsaw (1985) (in Polish)

Lemarchand, C., Couvreur, P., Vauthier, C., Costantini, D., Gref, R.: Study of emulsion stabilization by graft copolymers using the optical analyzer Turbiscan. Int. J. Pharm. 254, 77-82 (2003)

Nutt, C.W.: The physical basis of the displacement of oil from porous media by other fluids: a capillary bundle model. Proc. R. Soc. Lond. A 382, 155-178 (1982)

Redapangu, P.R., Sahu, K.C., Vanka, S.P.: Study of pressure-driven displacement flow of two immiscible liquids using a multiphase lattice Boltzmann approach. Phys. Fluids 24, 102-110 (2012)

Redapangu, P.R., Sahu, K.C., Vanka, S.P.: A lattice Boltzmann simulation of three-dimensional displacement flow of two immiscible liquids in a square duct. J Fluid Eng (TASME) 135, 121-202 (2013)

Sahu, K.C., Ding, H., Valluri, P., Matar, O.K.: Linear stability analysis and numerical simulation of miscible channel flows. Phys. Fluids 21, 042-104 (2009a)

Sahu, K.C., Ding, H., Valluri, P., Matar, O.K.: Pressure-driven miscible two-fluid channel flow with density gradients. Phys. Fluids 21, 043603 (2009b)

Sęk, J., Błaszczyk, M., Dziubiński, M., Padyk, A.: The study of permeation process of model fluids and fluids formed from the processing of crude oil through porous medium. Environ. Eng. Eco. Technol. 26, 48-58 (2011) (in Polish)

Taghavi, S.M., Alba, K., Seon, T., Wielage-Burchard, K., Martinez, D.M., Frigaard, I.A.: Miscible displacement flows in near-horizontal ducts at low Atwood number. J. Fluid Mech. 696, 175-214 (2012)

Talon, L., Meiburg, E.: Plane Poiseuille flow of miscible layers with different viscosities: instabilities in the Stokes flow regime. J. Fluid Mech. 686, 484-506 (2011)

Talon, L., Goyal, N., Meiburg, E.: Variable density and viscosity, miscible displacements in horizontal HeleShaw cells, part 1: linear stability analysis. J. Fluid. Mech. 721, 268-294 (2013)

Van Genuchten, M.Th.: A closed form equation for predicting the hydraulic conductivity of unsaturated soils. Soil Sci. Soc. Am. J. 44, 892-898 (1980)

Wang, J., Dong, M.: Trapping of the non-wetting phase in an interacting triangular tube bundle model. Chem. Eng. Sci. 66, 250-259 (2011)

Wielhorski, Y., Abdelwahed, A.B., Bizet, L., Bréard, J.: Theoretical and Experimental Modeling of Bubble Formation with Connected Capillaries in Liquid Composite Moulding Processes. Laboratoire Ondes et Milieux Complexes (LOMC), 540, France (2012). http://hal.archives-ouvertes.fr/docs/00/71/86/87/PDF/ FPCM11-paper-Wielhorski.pdf

Yan, Z., Elliott, J.A.W., Masliyah, J.H.: Roles of various bitumen components in the stability of water-indiluted-bitumen emulsions. J. Colloid Interface Sci. 220, 329-337 (1999)

Yang, D., Currier, R.P., Zhang, D.Z.: Ensemble phase averaged equations for multiphase flows in porous media. Int. J. Multiph. Flow 35, 628-639 (2009) 